\title{
Netrin-1 Induces Axon Branching in Developing Cortical Neurons by Frequency-Dependent Calcium Signaling Pathways
}

\author{
Fangjun Tang ${ }^{1}$ and Katherine Kalil ${ }^{1,2}$ \\ ${ }^{1}$ Neuroscience Training Program and ${ }^{2}$ Department of Anatomy, University of Wisconsin-Madison, Madison, Wisconsin 53706
}

\begin{abstract}
A single axon can innervate multiple targets by collateral branching. Axon branching is thus essential for establishing CNS connectivity. However, surprisingly little is known about the mechanisms by which branching is regulated. Axons often stop elongating before branches develop and anatomical and molecular data suggest that axon branching occurs independent of axon outgrowth. We found that netrin-1 dramatically increases cortical axon branching. Here, we sought to identify intracellular signaling components involved in netrin-1-induced axon branching. Using live cell imaging of dissociated developing cortical neurons, we show that netrin-1 rapidly increases the frequency of repetitive calcium transients. These transients are often restricted to small regions of the axon. Simultaneous imaging of calcium activity and development of axon branches revealed that $\mathrm{Ca}^{2+}$ transients coincide spatially and temporally with protrusion of branches from the axon. Remarkably, fully formed branches with motile growth cones could develop de novo within $20 \mathrm{~min}$. Netrin-1-induced $\mathrm{Ca}^{2+}$ transients involve release from intracellular stores and $\mathrm{Ca}^{2+}$ signaling is essential for netrin-1-induced axon branching. Using techniques to overexpress or suppress kinase activity, we find that calcium/calmodulin-dependent protein kinase II (CaMKII) and mitogen-activated protein kinase (MAPK) are major downstream targets of the netrin-1 calcium signaling pathway and are required for axon branching. CaMKII, but not MAPK, is also involved in axon outgrowth. The role of CaMKII and MAPKs in axon branching is consistent with the sensitivity of these kinases to changes in the frequency $\mathrm{Ca}^{2+}$ transients. Together, these novel findings define calcium signaling mechanisms required for development of new axon branches promoted by a guidance cue.
\end{abstract}

Key words: cortical development; axon guidance; netrin-1; axon branching; calcium transients; CaMKII

\section{Introduction}

Axons are guided toward targets by responses of their motile growth cones to environmental cues (Dickson, 2002). However, in the complex environment of the mammalian CNS, growth cones guide axons along pathways but do not typically extend into targets. Rather, axons extend collateral branches, and, in this way, a single axon can innervate multiple targets. Thus, axon branching is a fundamental mechanism for establishing connectivity in the developing CNS. Axon branching is also important in regenerative sprouting after injury (Schwab, 2002). During development, axons are known to stop elongating before branches begin to extend (O'Leary et al., 1990), and anatomical and molecular data suggest that axon branching may occur independent of axon outgrowth (Halloran and Kalil, 1994; Ng et al., 2002;

\footnotetext{
Received Dec. 7, 2004; revised June 10, 2005; accepted June 11, 2005.

This work was supported by National Institutes of Health Grant NS14428 and by a grant from the Whitehal Foundation. F.T. was supported in part by funds from the University of Wisconsin Graduate School and the University of Wisconsin Medical School. We thank the following for generously providing us with DNA constructs: Dr. Tobias Meyer for EGFP- $\alpha$ and $\beta$-CaMKII, Dr. Thomas Soderling for EGFP-CaMKIIN and constitutively active (CA) CaMKII (H282R), Dr. Azad Bonni for U6 and U6- $\alpha$-CaMKII RNAi vectors, Dr. Melanie Cobb for Erk2-MEK1 and Erk2-MEK1LA, Dr. Michael Weber for CA MEK1, and Dr. Alfred Wittinghofer for dominant-negative RasN17. We also thank Dr. Mary Halloran for comments on this manuscript.

Correspondence should be addressed to Katherine Kalil, Department of Anatomy, University of WisconsinMadison, 1300 University Avenue, Madison, WI 53706. E-mail: kakalil@facstaff.wisc.edu.

DOI:10.1523/JNEUROSCI.0871-05.2005

Copyright $\odot 2005$ Society for Neuroscience $\quad$ 0270-6474/05/256702-14\$15.00/0
}

Colavita and Tessier-Lavigne, 2003). Thus, for example, we found that netrin-1 increases cortical axon branching without affecting outgrowth of the primary axon (Dent et al., 2004). However, mechanisms regulating axon branching independent of axon outgrowth are poorly understood.

Axon branching is essential for establishing CNS connectivity, but surprisingly little is known about the mechanisms by which branching is regulated (Dent et al., 2003; Kornack and Giger, 2005). Calcium signaling is an important regulator of axon outgrowth and guidance (Gomez and Spitzer, 2000; Henley and Poo, 2004). Previously, we found that, in cortical neurons, spontaneous global $\mathrm{Ca}^{2+}$ transients regulate axon outgrowth in a frequency-dependent manner such that high-frequency repetitive transients were correlated with slowing of axon outgrowth, whereas low-frequency transients were associated with more rapid outgrowth (Tang et al., 2003). Recently, we found that application of netrin-1 induces extensive axon branching on cortical neurons (Dent et al., 2004). How does netrin-1 promote axon branching? Because netrin-1 has been shown to elevate $\mathrm{Ca}^{2+}$ levels in growth cones during steering events (Hong et al., 2000; Ming et al., 2002; Nishiyama et al., 2003), we hypothesized that netrin-1 may promote axon branching through $\mathrm{Ca}^{2+}$ changes in the axon. Moreover, because $\mathrm{Ca}^{2+}$ frequencydependent mechanisms are important in regulating cortical axon outgrowth (Tang et al., 2003), we hypothesized that repetitive 
$\mathrm{Ca}^{2+}$ transients may also play a role in netrin-1-induced axon branching. Activation of specific signaling components are known to be related to frequencies of $\mathrm{Ca}^{2+}$ transients. Calcium/ calmodulin-dependent kinase II (CaMKII) is known to function as a $\mathrm{Ca}^{2+}$ spike-frequency detector (Hudmon and Schulman, 2002), and mitogen-activated protein kinases (MAPKs) are also sensitive to intracellular $\mathrm{Ca}^{2+}$ changes (Cullen and Lockyer, 2002). Therefore, if repetitive $\mathrm{Ca}^{2+}$ transients are indeed involved in axon branching, CaMKII and MAPKs could be likely candidates as downstream targets of $\mathrm{Ca}^{2+}$ signaling. In this study, we sought to identify the intracellular signaling components involved in netrin-1-induced axon branching.

Here, we show for the first time that netrin-1 promotes rapid and extensive cortical axon branching by evoking repetitive $\mathrm{Ca}^{2+}$ transients in regions of the axon. Netrin-1 was also able to modulate endogenous $\mathrm{Ca}^{2+}$ activity by increasing the frequency of $\mathrm{Ca}^{2+}$ transients. We show that CaMKII and MAPK are major components of the netrin-1 calcium signaling pathway essential for axon branching and that CaMKII, but not MAPK, also plays a role in axon outgrowth. Together, these novel findings define mechanisms by which netrin-1 can promote a dramatic increase in axon branching in developing cortical neurons.

\section{Materials and Methods}

Cell culture. Dissociated cultures were prepared from sensorimotor cortices obtained from postnatal day 0 (P0)-P3 golden Syrian hamster $(\mathrm{Me}$ socricetus auratus), as described previously (Dent and Kalil, 2003). Neurons were cultured in dishes coated with $1.0 \mathrm{mg} / \mathrm{ml}$ poly-D-lysine at a density of $4-8 \mathrm{k} / \mathrm{cm}^{2}$ for calcium imaging and immunocytochemistry, $0.8 \mathrm{k} / \mathrm{cm}^{2}$ for netrin-1-induced branching experiments, and $15 \mathrm{k} / \mathrm{cm}^{2}$ for immunoblotting assay. Neurons in our cultures have already developed dendrites and a long axon with a growth cone at $15 \mathrm{~h}$ after plating (Szebenyi et al., 1998). At this stage, axons and dendrites can be clearly identified. Thus, we performed our calcium imaging experiments as early as $15 \mathrm{~h}$ after plating. In some experiments, when a $\mathrm{Ca}^{2+}$-free medium was required to test the necessity of extracellular $\mathrm{Ca}^{2+}$ for axon branching, Neurobasal medium was replaced by calcium-free DMEM medium in which cortical neurons survived well.

Experimental reagents. Recombinant chicken protein netrin-1 was purchased from R \& D Systems (Minneapolis, MN). Stock solutions were prepared by solubilizing drugs in water, dimethylsulfoxide (DMSO), or methanol according to the recommendations of the manufacturer. The following drugs in stock solutions were diluted in serum-free medium and bath applied to cultures: the L-type voltage-gated $\mathrm{Ca}^{2+}$ channel (VGCC) antagonist nifedipine (15 $\mu \mathrm{m}$; Calbiochem, La Jolla, CA), the ryanodine receptor antagonist dantrolene $(20 \mu \mathrm{m}$; Alomone Labs, Jerusalem, Israel), the inositol 1,4,5 trisphosphate $\left(\mathrm{IP}_{3}\right)$ receptor antagonist 2-aminoethoxydiphenyl borate $(100 \mu \mathrm{m}$; Calbiochem $)$, the general inhibitor to CaM kinases ( $5 \mu \mathrm{m}$ KN62; Calbiochem), the specific inhibitor to CaMKII (200 nM cell-permeable AIPII; Calbiochem), the MAPK inhibitors 1,4-diamino-2,3-dicyano-1,4-bis(o-aminophenylmercapto) butadiene (U0126; $10 \mu \mathrm{M}$; Cell Signaling Technology, Beverly, MA) and 2-(2-amino-3-methyoxyphenyl)-4 H-1-benzopyran-4-one (PD98059; $20 \mu \mathrm{M}$; Promega, Madison, WI). The antibody to $\alpha \mathrm{CaMKII}$ were obtained from Zymed Laboratories (South San Francisco, CA). The antibody against CaMKII, when phosphorylated at threonine 286, was purchased from Cell Signaling Technology and Promega. Phospho-MAP kinase kinase 1/2 (MEK1/2) and extracellular signal-regulated kinase 1/2 (Erk1/2) were all acquired from Cell Signaling.

DNA constructs, cell transfection, and image acquisition. DNA constructs were amplified by QIAfilter Plasmid Maxi kits (QIAGEN Sciences, Germantown, MD). Neurons at $1 \mathrm{~d}$ in vitro (DIV) were transfected with Lipofectamine 2000 (Invitrogen, Carlsbad, CA). Dishes were treated with $1 \mu \mathrm{g}$ of DNA and $1 \mu \mathrm{l}$ of Lipofectamine 2000 reagent in OptiMEM buffer (Invitrogen). Enhanced green fluorescent protein (EGFP) was cotransfected with non-GFP or myc-tagged plasmids. The cotransfection efficiency was monitored by immunostaining of EGFP-transfected cells with antibodies to proteins expressed by the plasmids and was found to reach $>90 \%$. Neurons with moderate levels of EGFP fluorescence developed typical dendritic and axonal process similar to nontransfected cells and remained healthy. At 3 DIV, cells were fixed and images of fluorescent neurons were acquired with a $40 \times / 1.0$ numerical aperture (NA) Plan Apo CF160 objective (Nikon, Tokyo, Japan). Neurons transfected with myc-tagged plasmids were subjected to immunostaining by antimyc antibody (Oncogene Research, San Diego, CA) before image acquisition. Montages of representative neurons were constructed using Adobe Photoshop CS (Adobe Systems, Mountain View, CA). The length of axons and branches and number of branches was measured with MetaMorph software (Universal Imaging, Downingtown, PA).

Local application of netrin-1. Gradients of netrin-1 applied to localized regions of axons and growth cones were established by locally puffing netrin-1 from a 0.5-1.0 $\mu \mathrm{m}$ pipette connected to an Eppendorf (Hamburg, Germany) microinjection system. The pipette was loaded with a stock solution of netrin-1 $(25 \mu \mathrm{g} / \mathrm{ml})$ and positioned $10-20 \mu \mathrm{m}$ from a growth cone or axon. Netrin-1 was ejected from the pipette tip by a pressure of 20-30 $\mathrm{kPa}$ every $1 \mathrm{~s}$ for 15-60 min during differential interference contrast (DIC) and fluorescence time-lapse imaging. The pipette loaded with a fluorescent dextran was used to demonstrate local gradients.

Time-lapse calcium and DIC imaging. Cortical neurons were loaded with membrane-permeable 2-4 $\mu \mathrm{m}$ Fluo-4 AM (Molecular Probes, Eugene, OR) predissolved in $0.01 \%$ pluronic acid (Molecular Probes) and $0.1 \%$ DMSO for $30 \mathrm{~min}$. Excess dye was washed out with three to five rinses of serum-free medium. In some experiments, when netrin-1 was bath applied, coverslips on which neurons were plated were enclosed in a chamber consisting of a $15 \mathrm{~mm}$ glass ring (Thomas Scientific, Swedesboro, NJ). In a few experiments, TTX was bath applied to neurons to eliminate spontaneous electrical activity. This allowed us to determine whether $\mathrm{Ca}^{2+}$ activity could be evoked by netrin-1 in neurons without ongoing $\mathrm{Ca}^{2+}$ transients. The dishes were returned to the incubator for $30-60$ min. Fluorescence imaging of intracellular $\mathrm{Ca}^{2+}$ dynamics in time-lapse for periods ranging from $10 \mathrm{~min}$ to $1 \mathrm{~h}$ was performed with a Nikon TE300 Quantum inverted epifluorescence microscope equipped with a Princeton Instruments (Trenton, NJ) MicroMax 512BFT cooled CCD camera containing a back-thinned, frame-transfer EEV CCD57-10 chip (Roper Scientific, Tucson, AZ). The imaging system was controlled by MetaMorph Software (Universal Imaging). Neurons were imaged in time lapse with either a $60 \times$ or a $100 \times 1.4$ NA Plan Apo CF160 objective (Nikon). Images were captured every 5-15 s, with 150-500 ms exposures, and under low light-level conditions achieved by illuminationattenuating neutral-density filters (Chroma Technology, Brattleboro, VT). Images were collected at a slow transfer rate, which reduces background noise, and binned $(2 \times 2)$. In some experiments, DIC images were taken in rapid succession with fluorescence $\mathrm{Ca}^{2+}$ imaging to determine the relationship between development of new branches and intracellular $\mathrm{Ca}^{2+}$ changes. Alternate acquisition of both DIC and fluorescence images were automatically controlled by a Lambda 10-2 dual-filter wheel (Sutter Instruments, Novato, CA).

Measurement and analysis of $\mathrm{Ca}^{2+}$ transients. Measurements of transients in growth cones and axons were obtained as described previously (Tang et al., 2003). Briefly, average fluorescent pixel intensity of the region of interest in a growth cone or an axon was digitally quantified with MetaMorph software, subtracted from background, and normalized to baseline fluorescence intensity. Fluorescence increases $>150 \%$ of baseline were generally characterized as $\mathrm{Ca}^{2+}$ transients, which were further confirmed by frame-by-frame examination of the time-lapse movie.

Quantification of axon branches and image processing. In experiments with long-term treatments, $100 \mathrm{ng} / \mathrm{ml}$ netrin-1 and other reagents were bath applied to cultures $7-10 \mathrm{~h}$ after plating. At 3 DIV, cultures were fixed, and images were acquired with a $10 \times / 0.5 \mathrm{NA}$ Neofluar phasecontrast objective on a Zeiss (Thornwood, NY) 35M inverted microscope. Processes extending $>20 \mu \mathrm{m}$ at orthogonal angles to the axon were characterized as branches. We counted only the number and length of branches extending from primary axons. Each individual experiment was repeated at least three times. Images shown in the figures were enhanced using the unsharp mask filter, high pass, and brightness-contrast 
adjustment functions in Adobe Photoshop. Time-lapse images were assembled into QuickTime movies (Premiere; Adobe Systems). Graphs were created in SigmaPlot (SPSS, Chicago, IL). Images were processed with MetaMorph 4.62 and all figures were constructed in Photoshop CS (Adobe Systems).

Statistical analysis. Statistical analyses of control and experimental groups were performed using Sigmastat (Jandel Scientific, Corte Madera, CA). Significant difference between control neurons and those in a single experimental condition was determined by Student's $t$ test. Multiple experimental groups were compared with controls with the Krustal-Wallis one-way ANOVA on ranks with Dunn's post hoc test. In pharmacological experiments in which cortical neurons were treated with various drugs for $3 \mathrm{~d}$, at least 60 neurons in each experimental condition were chosen for measurements of branch number and length of axons and branches. In experiments in which overexpression of different constructs of CaMKII and MAPK were used, at least 30 transfected neurons in each experimental condition were used for measurements of branch number and length of axons and branches. Each experiment was repeated at least three different times. In experiments using time-lapse microscopic imaging, we tested the effects of local repetitive application of netrin-1 on 30 axons, of which 15 formed new branches within $1 \mathrm{~h}$ in response to netrin-1. In contrast, in control experiments $(n>10)$ in which $0.5 \%$ BSA in PBS buffer was locally applied to cortical axons, localized branch formation on axons rarely occurred. In four separate experiments, gradients of netrin-1 locally applied to cortical axons induced transient filopodial activity, which disappeared after withdrawal of netrin.

Immunocytochemistry. At 15-60 min after addition of netrin-1 to cortical neurons at 2 or 3 DIV, neurons were fixed and immunostained as described previously (Dent and Kalil, 2001). Primary antibodies (phospho-CaMKII and phospho-MAPK) from both Cell Signaling Technology and Promega and secondary antibodies from Jackson ImmunoResearch (West Grove, PA) were used to detect phosphorylation of CaMKII and MAPK. In some experiments, to quantify and compare phosphorylation of CaMKII or MAPK at different times after stimulation of netrin-1, the same cultures were fixed and processed with first and secondary antibodies at the same time. Images were taken on the same day and under the same conditions, including exposure time, illumination, and digital scaling. In a few experiments, to determine the relationship between netrin-induced calcium activity and phosphorylation of CaMKII, we first performed time-lapse calcium imaging for 10-15 min and rapidly fixed the cultures on the microscope stage, followed by immunocytochemistry. In these experiments, we used dishes attached with etched grid glass coverslips to provide landmarks for relocating the cells (Bellco Glass, Vineland, NJ).

Western blotting. Neurons at 3 DIV were treated with $100 \mathrm{ng} / \mathrm{ml}$ netrin-1 or $50 \mathrm{~mm} \mathrm{KCl}$, solubilized in $60 \mu \mathrm{l}$ of ice-cold extraction buffer [50 mм Tris, pH 8.0, $150 \mathrm{~mm} \mathrm{NaCl}, 50 \mathrm{~mm}$ sodium fluoride, 5 mм EDTA, $0.5 \%$ sodium deoxycholate, $1 \%$ IGEPAL CA-630, $10 \mathrm{mg} / \mathrm{ml}$ PMSF, 100 mM sodium vanadate (all from Sigma, St. Louis, MO), and complete protease inhibitor cocktail tablet (Roche Diagnostics, Indianapolis, IN)], and centrifuged at $14,000 \mathrm{rpm}$ for $5 \mathrm{~min}$. Supernatants were absorbed for immunoblotting with rabbit antibodies to phosphorylated forms of CaMKII (Promega) and protein kinase C (PKC) (Cell Signaling Technology). Blots were developed in ECL + (Amersham Biosciences, Arlington Heights, IL).

\section{Results}

\section{Netrin-1 induces rapid axon branching}

Previously (Dent et al., 2004), we found that bath application of netrin-1 to cortical neurons over several days promotes extensive axon branching, but not axon elongation, and that local application induces rapid localized axon branching. Here, to investigate the time course of branching, we bath applied netrin-1 (100 ng/ $\mathrm{ml}$ ) to cortical cultures for $3 \mathrm{~d}$, which increased axon branching by more than threefold, consistent with our previous findings. Untreated axons typically have only a few branches, whereas netrin-1-treated axons had elaborate multibranched arbors $(n=$ 176) (Fig. $1 A$ ) with $\sim 40 \%$ increase in branch length. Within $1 \mathrm{~h}$ of netrin-1 treatment, axons had developed branches up to 22 $\mu \mathrm{m}$ in length $(n=8)$ (Fig. $1 B, C)$, whereas controls did not develop significant branching until several days in culture. Branches on netrin-1-treated neurons could develop from growth cone filopodia that thickened, elongated, and elaborated a growth cone (Fig. $1 B$ ). Branches also developed from axonal filopodial processes that elongated and branched again into arbors (Fig. 1C). Local application of netrin-1 pulsed through a glass pipette positioned $25 \mu \mathrm{m}$ from the axon elicited new branches within a few minutes in $\sim 50 \%$ of axons tested $(n=30)$. In close proximity to the pipette tip, branches developed from preexisting filopodia (Fig. 1D), de novo from smooth regions of the axon (Fig. $1 E$ ), or from increased lammellipodia along the axon shaft (Fig. $1 F)$. In several cases $(n=4)$, netrin- 1 elicited transient filopodia (Fig. 1G) for 16 min that disappeared within 16 min after the pipette was withdrawn. Thereafter, filopodial activity did not change in the absence of netrin-1. These results suggest that netrin-1 can induce rapid branching on responsive neurons within 5 min by either additional growth of existing filopodia or de novo protrusions from the axon shaft. Moreover, netrin-1 is required for the maintenance of developing branches.

\section{Netrin-1 induces repetitive calcium transients in cortical axons}

To determine the effects of netrin- 1 on $\mathrm{Ca}^{2+}$ activity in cortical neurons, we bath or locally applied netrin-1 and measured changes in intracellular $\mathrm{Ca}^{2+}$ with the calcium indicator Fluo-4. Application of netrin-1 evoked increases in $\mathrm{Ca}^{2+}$ activity within minutes in $59.4 \%$ of neurons tested $(n=128)$. In $32 \%$ of neurons, there were no detectable changes in activity, and, in $8.6 \%$ of neurons, calcium activity decreased. Within a few minutes, netrin-1 elicited repetitive $\mathrm{Ca}^{2+}$ transients in neurons showing no previous activity (Fig. $2 A$ ) or increased the frequency and amplitude of endogenous $\mathrm{Ca}^{2+}$ transients (Fig. $2 \mathrm{~B}$ ). In a few experiments, we used TTX to silence endogenous $\mathrm{Ca}^{2+}$ transients. We found that netrin- 1 could evoke $\mathrm{Ca}^{2+}$ transients in neurons showing no endogenous activity (data not shown). BSA (15 control experiments) had no effect on calcium transients (Fig. 2C). Increased frequency was the most common type of change in $\mathrm{Ca}^{2+}$ transients after netrin-1 application. Measurements of calcium changes in 32 neurons responsive to netrin-1 showed that the average frequency of $\mathrm{Ca}^{2+}$ transients was increased fourfold ( $t$ test $p<001$ ) from 0.2 to 0.8 per minute (Fig. $2 D)$. Netrin-1 application could elicit localized $\mathrm{Ca}^{2+}$ changes that spread globally along the entire axon (Fig. 2E1-E7), or such changes could remain restricted to small regions of the axon shaft averaging $15.3 \mu \mathrm{m}$ in length (Fig. 2F1-F4). Netrin-1-evoked $\mathrm{Ca}^{2+}$ transients in an axon and its branches often occurred in patterns, frequencies, and amplitudes that were different and distinct in the axon and its branches (Fig. 2G1-G7) (supplemental movie, available at www.jneurosci.org as supplemental material). Changes in $\mathrm{Ca}^{2+}$ transients were maintained for the entire observation period (5-30 min), but when netrin-1 was withdrawn, $\mathrm{Ca}^{2+}$ activity gradually declined within minutes to baseline levels (data not shown).

\section{Calcium activity is required for axon branching}

Subsequently, we asked whether netrin-1-induced branching requires $\mathrm{Ca}^{2+}$ signaling. First, we sought to determine the source of netrin-1-induced $\mathrm{Ca}^{2+}$ activity. Cortical axon elongation is regulated by calcium entry through voltage gated L-type channels (Tang et al., 2003). To determine whether this mode of calcium entry is also involved in netrin-1-induced activity, we applied the L-type 

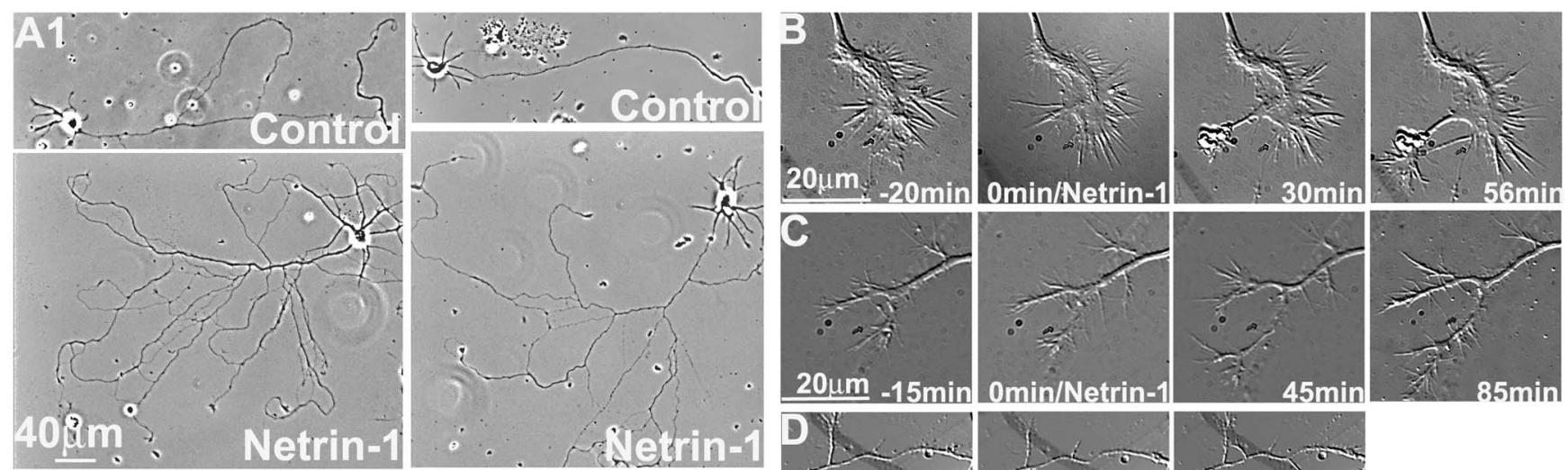

\section{D}
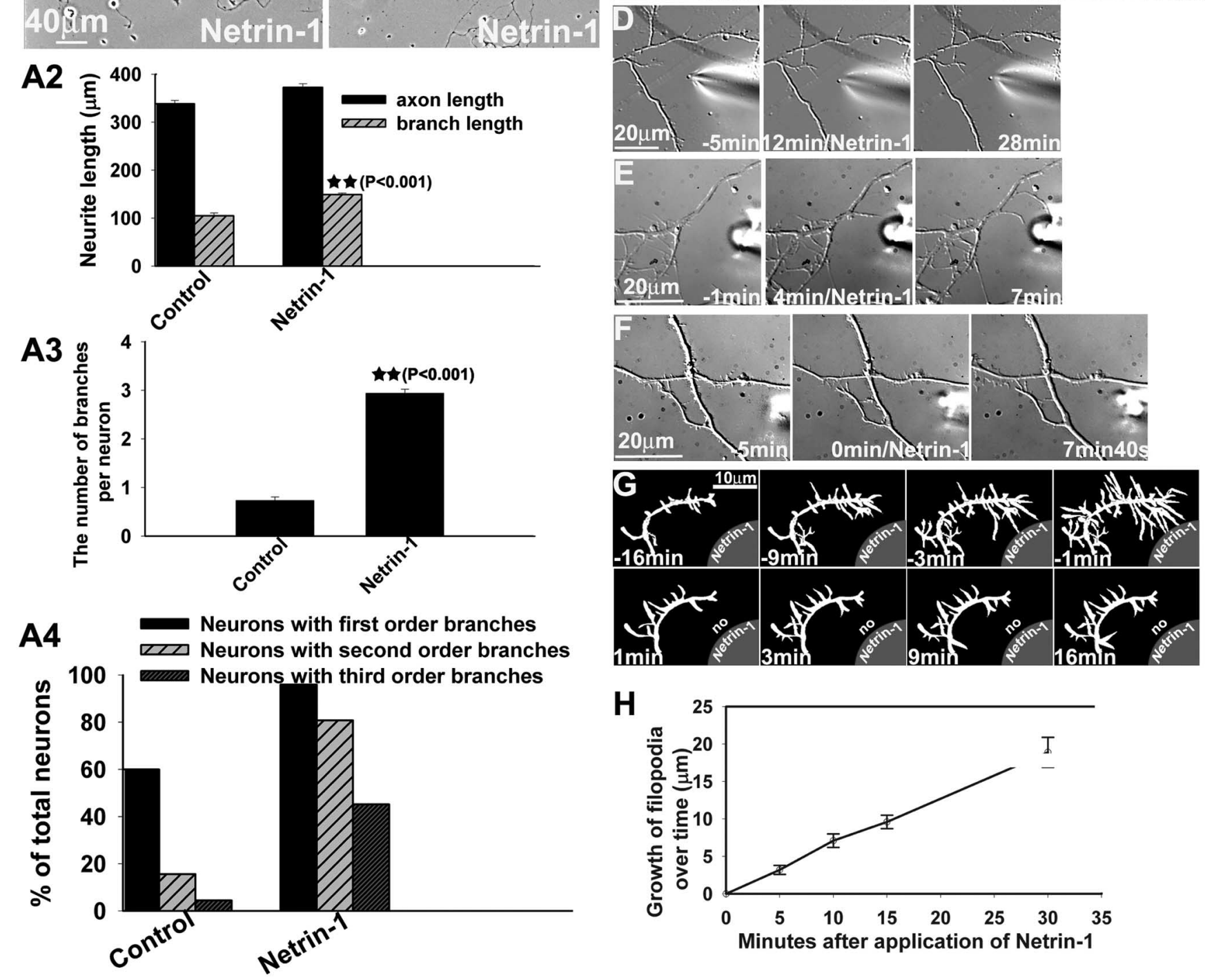

Figure 1. Bath-applied netrin-1 increases axon branching without affecting axon length, and locally applied netrin-1 induces filopodial protrusions on the axon shaft. A1, Examples of cortical neurons treated for $3 \mathrm{~d}$ with BSA (controls) and for $3 \mathrm{~d}$ with bath-applied netrin-1 $(100 \mathrm{ng} / \mathrm{ml})$ showing dramatic increase in axon branching with netrin-1. A2-A4, Bar graphs showing no increase in axon length but a significant increase in branch length (A2), a threefold increase in numbers of branches (A3), and a large increase in neurons with higher-order axon branches (A4) after netrin-1 treatment for 3 d. $\boldsymbol{B}, \boldsymbol{C}$, Sequences of time-lapse DIC images showing rapid development of axon branches after bath-applied netrin-1. $\boldsymbol{B}, \boldsymbol{C}$, An example of a branch extending from a growth cone and developing its own growth cone $(\boldsymbol{B})$ and increased axon branching by filopodial protrusions $(\boldsymbol{C})$. D-F, Locally applied netrin-1 induces rapid protrusion of filopodia $(\boldsymbol{D}, \boldsymbol{E})$ and lammelipodia $(\boldsymbol{F})$ on the axon in the region of the pipette tip. G, Series of tracings of DIC images showing extension of transient filopodia during 15 min time period when netrin- 1 is locally pulsed every $15 \mathrm{~s}$ (at top). Transient filopodia are illustrated as cumulative over time. When netrin- 1 is withdrawn (bottom), filopodial protrusions cease. $\boldsymbol{H}$, Graph plotting average growth of 12 axonal filopodia induced de novo from 10 neurons by netrin-1. Filopodia extend to $20 \mu \mathrm{m}$ over $30 \mathrm{~min}$.

channel blocker nifedipine and found in all cases $(n=7)$ that this did not interfere with the ability of netrin- 1 to induce robust $\mathrm{Ca}^{2+}$ transients (Fig. 3A). To determine the involvement of $\mathrm{Ca}^{2+}$ release from intracellular stores, we applied blockers to $\mathrm{IP}_{3}(n=6)$ and ryanodine receptors $(n=6)$ and found that, in all cases, $\mathrm{Ca}^{2+}$ transients were gradually attenuated (Fig. $3 B, C$ ). Thus, netrin-1-induced $\mathrm{Ca}^{2+}$ transients involve release from intracellular stores rather than $\mathrm{Ca}^{2+}$ entry through L-type channels. 

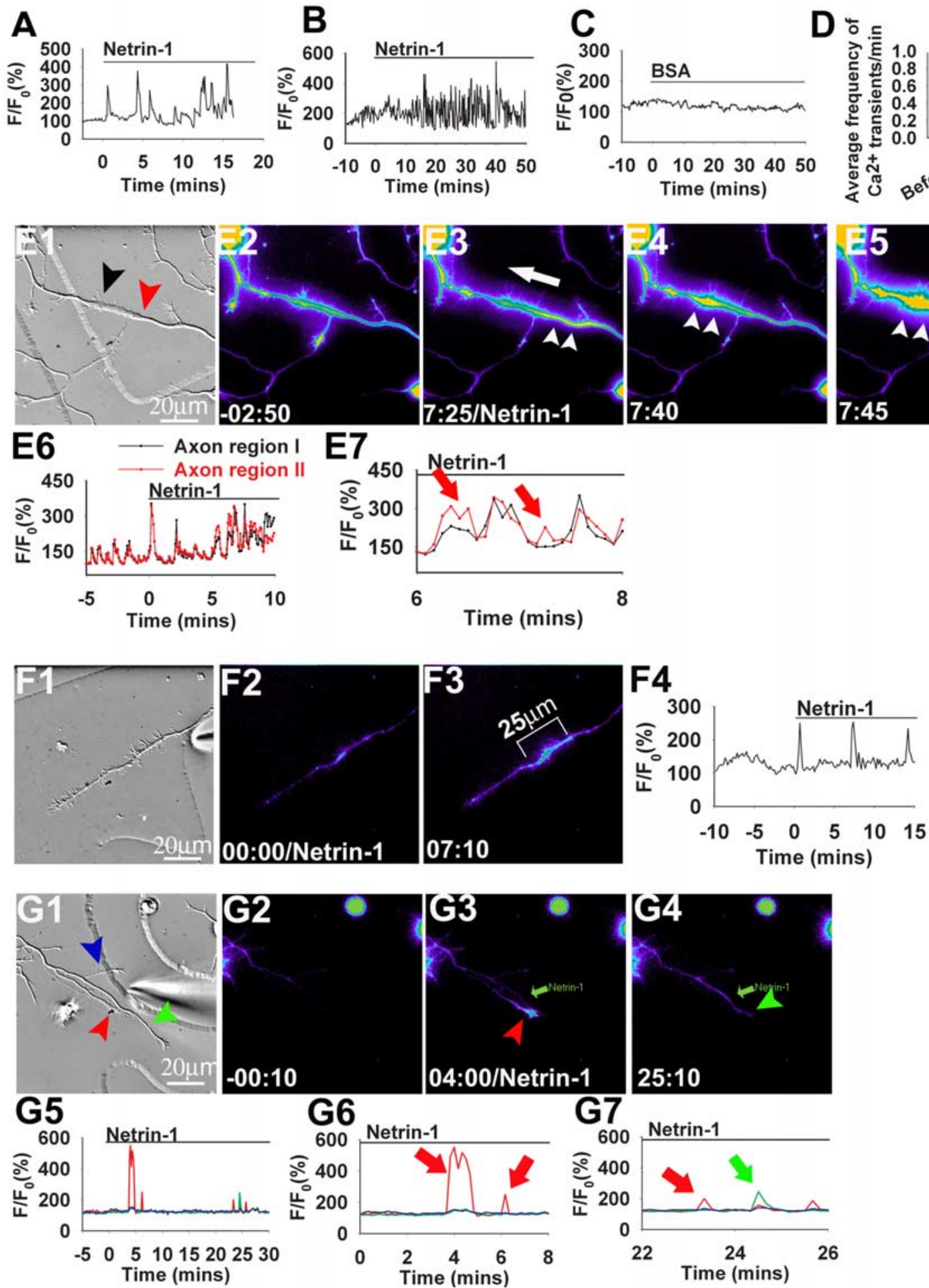

Figure 2. Repetitive calcium transients in cortical axons are evoked by netrin-1 application within minutes. $\boldsymbol{A}$, Netrin-1 evokes calcium activity in a previously silent axon. $\boldsymbol{B}$, Netrin-1 increases the frequency and amplitude of calcium transients. $C$, BSA (control) elicits no calcium changes. Changes in fluorescence intensity are shown relative to baseline $\left(F / F_{0} \%\right)$, and measurements of calcium changes in all examples were obtained from axons and their growth cones. $\boldsymbol{D}$, Graph comparing average frequency of calcium transients in 32 neurons before and after netrin-1 application. Frequency of calcium transients increases fourfold after netrin-1 application. E1-E5, DIC image (E1) and time-lapse images in pseudocolor (E2-E5), showing changes in calcium activity in a region of an axon after application of netrin-1. Calcium images were acquired every $5 \mathrm{~s}$. $\mathbf{E 3}$, A calcium transient begins in the region of the arrowheads and is propagated along the axon in the direction indicated by the arrow. E2-E5, Regions of high calcium activity correspond to the region of the red and black arrowheads shown in the DIC image in E1. Measurements of frequencies and amplitudes of calcium transients in the two axon segments corresponding to the red (region I) and black (region II) arrowheads are shown in $\mathbf{E} 6$ and magnified in $\boldsymbol{E 7}$ for the time period of 6 - 8 min. Red arrows indicate time points when peak amplitudes occur only in axon region I. F1-F4, Localized pulsed application of netrin-1 can evoke calcium repetitive calcium transients in small localized regions of the axon. F4, Changes in frequency of calcium transients in the region of the axon bracketed in F3. G1-G4, DIC image (G1) and time-lapse images in pseudocolor (G2-G4) showing changes in calcium activity in an axon (green arrowhead) and its two branches (blue and red arrowheads) after local application of netrin- 1 indicated by the green arrow. Localized calcium transients occur in one branch at $4 \mathrm{~min}$ (G3) and in the primary axon at $25 \mathrm{~min}$ (G4). Measurements of calcium transients in the axon (green) and in the branch (red) are shown during different time periods (G5-G7). Local transients in the branch are indicated by red arrows and are indicated in the primary axon by green arrow (see supplemental movie 1, available at www.jneurosci.org as supplemental material). As shown in G5-G7, calcium transients induced by netrin-1 can occur at different frequencies and asynchronously in an axon and its branches. 


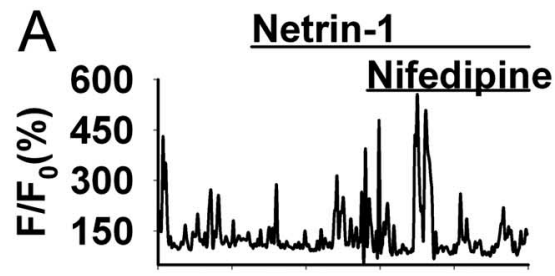

$\begin{array}{llllll}0 & 10 & 20 & 30 & 40 & 50\end{array}$ Time (mins)
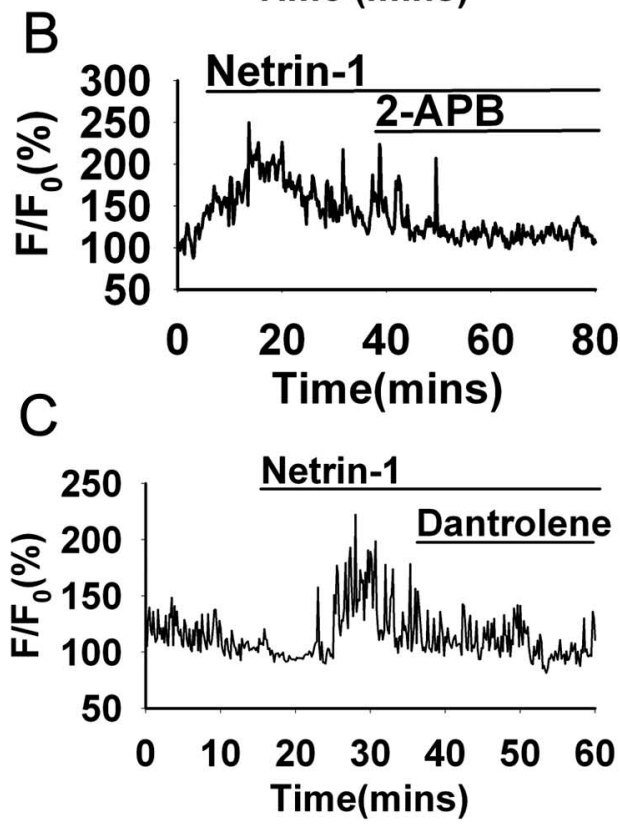
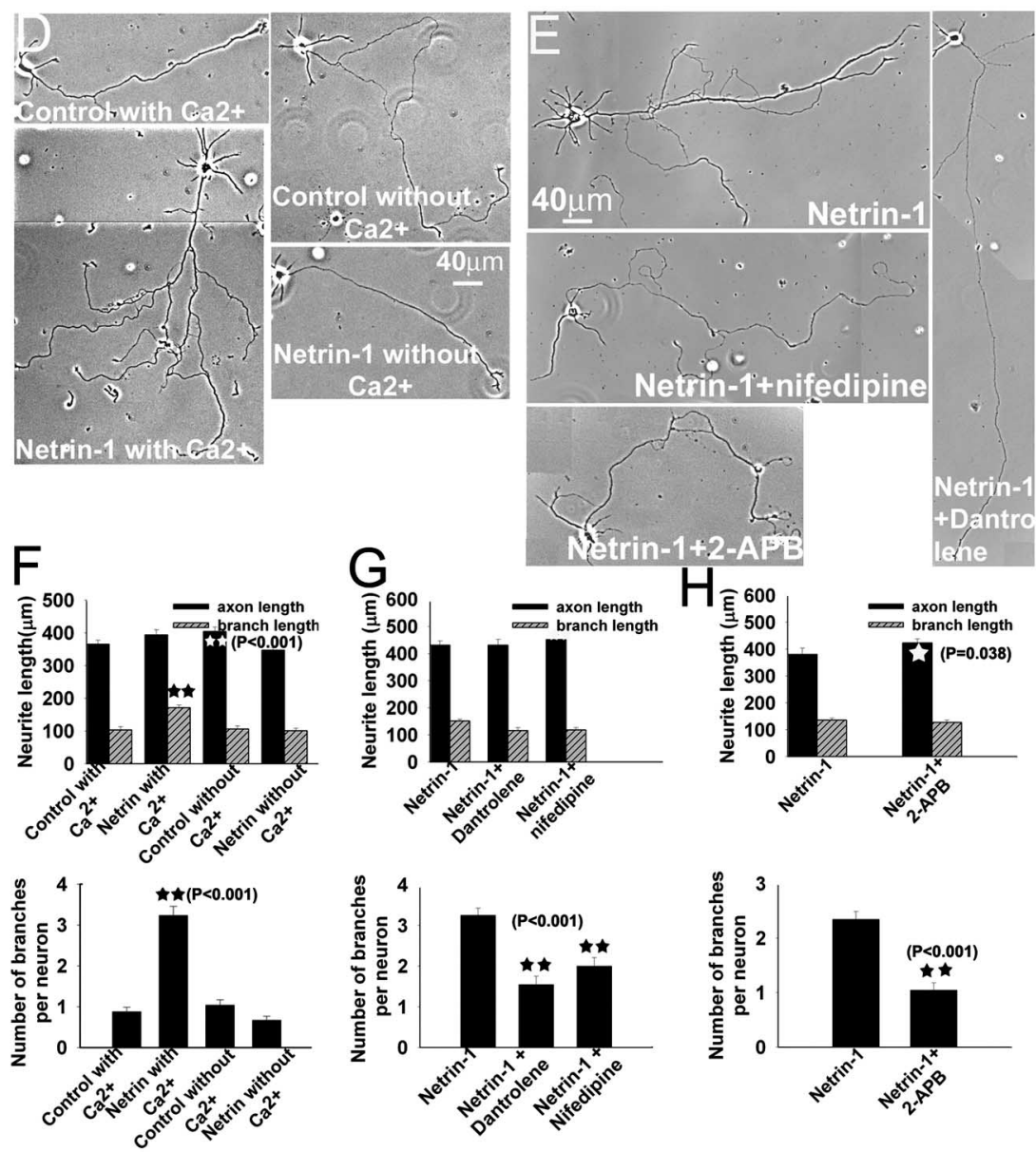

Figure 3. Calcium activity evoked by netrin- 1 involves release from intracellular stores and is required for axon branching. $\boldsymbol{A}-\boldsymbol{C}$, Examples of neurons in the presence of netrin-1 treated with nifedipine to block L-type voltage-gated channels $(\boldsymbol{A})$, 2-aminoethoxydiphenyl borate (2-APB) to block IP, receptors $(\boldsymbol{B})$, and dantrolene to block ryanodine receptors $(\boldsymbol{C})$. Neurons treated with 2-APB and dantrolene, but not nifedipine, show attenuation of calcium activity over time. $\boldsymbol{D}$, Examples of neurons cultured with and without calcium in the medium in the presence of $0.5 \%$ BSA (control) or $100 \mathrm{ng} / \mathrm{ml}$ netrin-1. In the absence of extracellular calcium, netrin-1 does not promote axon branching, but axons are slightly longer. $\boldsymbol{E}$, Examples of neurons treated with netrin-1 or with netrin-1 and the addition of nifedipine, 2-APB, or dantrolene, demonstrating that blocking calcium release from intracellular stores diminishes axon branching. $\boldsymbol{F}$ - $\boldsymbol{H}$, Bar graphs comparing axon length, branch length, and numbers of branches per neuron in all experimental conditions shown in the examples in $\boldsymbol{D}$ and $\boldsymbol{E}$.

We then measured axon branching under various conditions in which calcium activity was experimentally reduced. When cortical neurons were cultured in $\mathrm{Ca}^{2+}$ free medium (Fig. $3 D, F$ ), axon branching was similar to control levels without netrin-1, although axons were significantly longer. Importantly, netrin-1 applied to neurons in the absence of extracellular $\mathrm{Ca}^{2+}$ failed to elicit axon branching above control levels. In the subsequent set of experiments, in which calcium was present in the medium, we examined the source of calcium involved in netrin-1-induced axon branching. We found that application of the L-type channel blocker nifedipine partially suppressed netrin-1-induced axon branching, which is surprising in view of the result that nifedipine has no effect on netrin-1-induced $\mathrm{Ca}^{2+}$ transients (Fig. $3 E, G$ ). One possibility to explain this result is that netrin-induced calcium transients normally activate L-type channels that act on downstream kinases (Dolmetsch et al., 2001) to promote branching. When L-type channels are blocked, these signaling pathways are inactivated, and branching is reduced in the presence of calcium transients that could arise from entry through other types of channels. When $\mathrm{Ca}^{2+}$ release from intracellular stores was prevented by blockers to $\mathrm{IP}_{3}$ and ryanodine receptors, we found that axon branching was severely inhibited (Fig. $3 G, H$ ). These results show that $\mathrm{Ca}^{2+}$ signaling is required for netrin-1-induced axon branching and that release from intracellular stores is a major source of this $\mathrm{Ca}^{2+}$ activity. Nevertheless, we cannot rule out the possibility that calcium entry through L-type channels could contribute to netrin-1-induced axon branching.

To further demonstrate the importance of $\mathrm{Ca}^{2+}$ signaling in axon branching, we imaged netrin-1-induced branching events simultaneously with live cell $\mathrm{Ca}^{2+}$ imaging (Fig. 4). In four sequences lasting up to $30 \mathrm{~min}$, we were able to capture axon branching events that precisely coincided spatially and temporally with induction of $\mathrm{Ca}^{2+}$ transients. In one sequence, for example, application of netrin-1 to a localized region of the axon almost immediately elicited large $\mathrm{Ca}^{2+}$ changes along a $30 \mu \mathrm{m}$ length of the axon from which a $20 \mu \mathrm{m}$ branch subsequently developed (Fig. 4). $\mathrm{Ca}^{2+}$ transients in this region of the axon shaft greatly increased in frequency and amplitude over the subsequent 20 min during continuous application of netrin-1 (Fig. 4C). Remarkably, by $12 \mathrm{~min}$ a branch began to protrude de novo from the region of high calcium activity. By $20 \mathrm{~min}$, a motile growth cone had elaborated a dynamic arbor on which filopodia extended and retracted (supplemental movie, available at www.jneurosci.org as supplemental material). Thus, the axonal processes that developed in regions of high $\mathrm{Ca}^{2+}$ activity were not transient filopodia but stable arborizing branches with motile growth cones that 
persisted throughout the observation period. Several other branches also developed de novo from this region of the axon within $20 \mathrm{~min}$. Interestingly, the single long branch already present on the axon also showed localized high $\mathrm{Ca}^{2+}$ activity in a region from which numerous transient lamellar and filopodial processes protruded. These observations show that netrin-1 stimulation can induce high levels of $\mathrm{Ca}^{2+}$ transients on restricted regions of an axon coincident with rapid development of branches.

\section{CaMKII is involved in calcium}

signaling, regulating axon outgrowth and branching

We were interested in the downstream targets of netrin-1-induced $\mathrm{Ca}^{2+}$ signaling involved in axon branching. Because we found that the major effect of netrin-1 on $\mathrm{Ca}^{2+}$ transients was to increase their frequency, one likely downstream target is CaMKII, which is known to function as a detector of $\mathrm{Ca}^{2+}$ spike frequency (Hudmon and Schulman, 2002; Soderling et al., 2001). To determine whether netrin-1 activates CaMKII, we stained neurons with antibodies to phosphorylated CaMKII after treatment with either $\mathrm{KCl}$ or netrin-1, both of which increase intracellular $\mathrm{Ca}^{2+}$. Activation of CaMKII occurred within 15 min and persisted up to $1 \mathrm{~h}$ (Fig. $5 A, B$ ). Importantly, we performed live cell imaging of $\mathrm{Ca}^{2+}$ transients in neurons before and after netrin-1 treatment. We then rapidly fixed these cells and stained them with phospho-CaMKII antibodies. These experiments (Fig. $5 C$ ) revealed that only cells in which repetitive $\mathrm{Ca}^{2+}$ transients were induced by netrin-1 had strong phosphoCaMKII staining, demonstrating a strong correlation between increased frequency of $\mathrm{Ca}^{2+}$ transients and activation of CaMKII. In the subsequent set of experiments, we applied the CaMKII inhibitor KN62, which can inhibit several CaM-kinases, and AIPII which has been shown to be a specific inhibitor for CaMKII (Jourdain et al., 2003). KN62 completely abolished axon branching in the presence of netrin-1 and significantly reduced axon outgrowth (Fig. $6 A-C$ ). Although a previous study showed that CaMKII is necessary for $\mathrm{KCl}$-induced survival of sensory neurons (Vaillant et al., 1999), we found no adverse effects on neuron survival after treatment with CaMKII inhibitors. As shown in Figure 6, axon and dendritic outgrowth are robust even after $3 \mathrm{~d}$ of exposure to these inhibitors which selectively affect axon length and branching. AIPII treatment dramatically reduced numbers of axon branches, which were significantly shorter and lacking in higher-order branches (Fig. 6D-G) compared with netrin-1-stimulated neurons. Similar to KN62, AIPII also reduced axon outgrowth. These pharmacological experiments were performed by adding the CaMKII inhibitors to the cultures along with netrin-1 before branches had developed. To determine whether CaMKII is important for initiation or maintenance of axon branches we added the inhibitors $2 \mathrm{~d}$ after netrin-1 treatment when most branches had already formed.
Branching was only slightly reduced, suggesting that CaMKII is important for the initiation and growth of branches rather than their maintenance (data not shown). Together, these results suggest that CaMKII is involved in the netrin-1 signaling pathway underlying axon branching and also plays a role in axon outgrowth.

To provide additional evidence for the importance of CaMKII in axon branching, we transfected cortical neurons with several wild-type and mutant CaMKII constructs fused to EGFP. CaMKII is normally expressed in cortical neurons by $1 \mathrm{~d}$ in culture and increases over the next several days, during which axon branches are also developing (data not shown). Netrin-1 application increased CaMKII expression over this $3 \mathrm{~d}$ period (data not shown). We transfected neurons after $1 \mathrm{~d}$ in culture with either EGFP wild-type $\alpha$ CaMKII or $\beta$ CaMKII. Overexpression of $\alpha$ CaMKII but not $\beta$ CaMKII promoted a more than threefold increase in axon branching (Fig. 7A-D), and the morphologies of axon arbors were similar to those after netrin-1 treatment. Axon length was also significantly increased. Transfection with an autophosphorylation mutant of CaMKII (Fig. 7D) resulted in neurons with branching above control levels but still significantly less than in neurons transfected with wild-type CaMKII. This could mean that autophosphorylation is not responsible for $100 \%$ of the activity of CaMKII during netrin-1-induced axon branching. Transfection of neurons with constitutively active $\alpha$ CaMKII sim- 
A

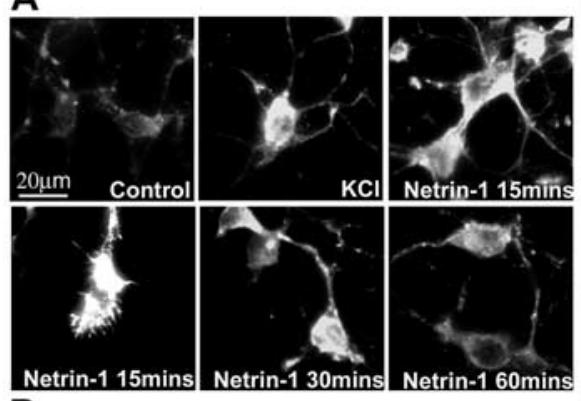

B

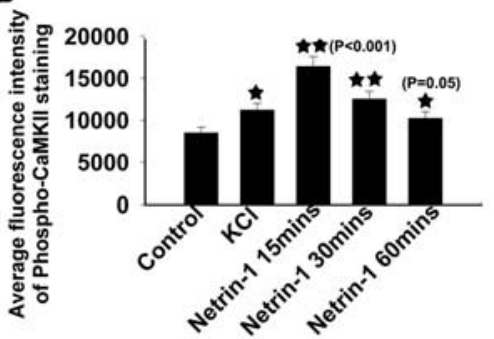

C
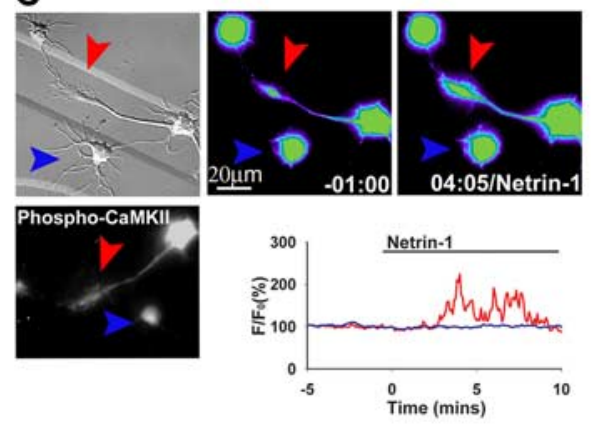

D

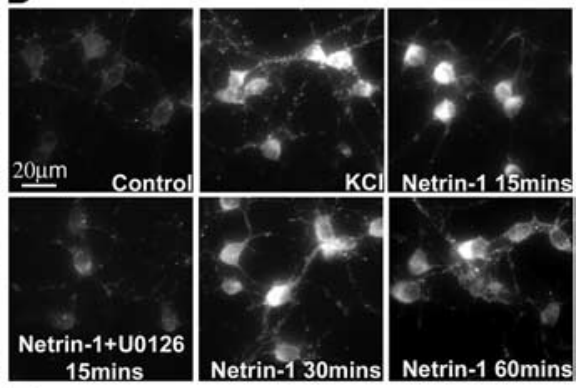

$\mathrm{E}$

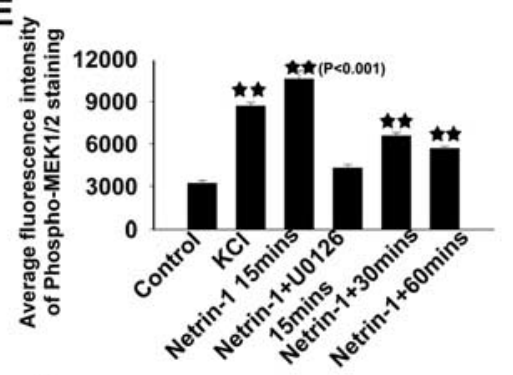

$\mathbf{F}$

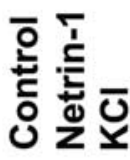

\section{- - Phospho-CaMKII \\ - - Syntaxin}

Figure 5. Netrin-1 activates both CaMKII and MAPK. $\boldsymbol{A}$, Examples of neurons treated with $0.5 \% \mathrm{BSA}$ (control) for $15 \mathrm{~min}, \mathrm{KCl}$ for 5 min, or netrin-1 for 15,30 , or 60 min and then immunostained with an antibody to CaMKII phosphorylated at the site of threonine 286. $\boldsymbol{B}$, Bar graphs comparing average fluorescence intensity of immunostained neurons subjected to treatments described in $\boldsymbol{A}$. C, Examples of neurons (shown in DIC) imaged first for 15 min to measure calcium activity after application of netrin-1, followed by rapid fixation and immunostaining of phospho-CaMKII. Intense staining only occurred in neurons showing repetitive netrin-1-induced calcium transients (red arrowhead points to growth cone of neuron with high calcium activity) but not in neurons that failed to show calcium activity in response to netrin-1 (blue arrow). Measurements of calcium activity were obtained from neurons indicated by red and blue arrowheads. D, Examples of neurons treated with $0.5 \%$ BSA (control) for 15 min, KCl for 5 min, netrin- 1 for 15 min with or without the MAPK inhibitor U0126, or netrin-1 for 30 or 60 min followed by immunostaining with an antibody to the phosphorylated MAPK, MEK1/2. $\boldsymbol{E}$, Bar graphs comparing average fluorescence intensity of immunostained neurons subjected to treatments described in $\boldsymbol{B}$. $\boldsymbol{F}$, Western blot showing increases in phosphorylated CaMKII after application of netrin-1 for $15 \mathrm{~min}$ or $\mathrm{KCl}$ for $5 \mathrm{~min}$. Syntaxin was used to control for loading of the same amount of protein sample.

ilarly increased axon length and branching (Fig. 7F). We also overexpressed a naturally occurring CaMKII inhibitory protein CaMKIIN. This protein has been well characterized with respect to its specificity in inhibiting CaMKII (Chang et al., 1998). CaMKIIN reduced axon branching to below control levels (Fig. $7 G-I$ ) and also resulted in shorter axons. In additional experiments, we examined effects of CaMKII overexpression in the presence of pharmacological inhibitors and found that branching was almost completely abolished (Fig. $7 J-P$ ). In a final set of experiments, we used several approaches to test the effects of inhibiting CaMKII on netrin-1-induced axon branching. First, we transfected neurons with the inhibitory protein CaMKIIN. Overexpression of CaMKIIN prevented netrin-1-induced axon branching but did not reduce axon length (Fig. $8 A-E$ ). In a second approach, we used an $\alpha$ CaMKII DNA vector-based RNA interference (RNAi) knockdown strategy (Sui et al., 2002; Gaud- illiere et al., 2004) to reduce expression of $\alpha$ CaMKII (Fig. 8F-G). Neurons transfected with the U6/ $\alpha$ CaMKII construct showed $\sim 50 \%$ reduction in $\alpha$ CaMKII fluorescence immunostaining (Fig. $8 G, H$ ), and neurons with reduced levels of $\alpha$ CaMKII showed no netrin-1-induced branching. In comparison, neurons transfected with a control vector U6 developed normal robust branching in the presence of netrin-1 (Fig. 8F3). These results demonstrate that when $\alpha$ CaMKII is suppressed, netrin-1 fails to induce axon branching. Together, these results provide compelling evidence that $\alpha$ CaMKII plays a central role in promoting axon branching through netrin-1-evoked calcium signaling.

MAPK is involved in calcium signaling underlying axon branching

MAPK signaling cascades (including Ras, MEK1/2, and Erk1/2) are also known to be sensitive to intracellular $\mathrm{Ca}^{2+}$ changes. We therefore investigated the possible role of MAPK in regulation of axon branching. As with CaMKII, netrin-1 led to rapid phosphorylation of MAPK (Fig. 5D,E). Application of the pharmacological inhibitors U0126 and PD98059 specific to MAPK resulted in a dramatic reduction in axon branching compared with netrin-1induced branching (Fig. 9A-C). Surprisingly, axon outgrowth was not affected, and axons were actually slightly longer than those treated with netrin-1. However, simultaneous application of CaMKII and MAPK inhibitors abolished axon branching and reduced axon outgrowth. Overexpression of constitutively active constructs of the MAPKs elicited extensive axon branching compared with EGFPtransfected controls (Fig. 9D-M). Conversely, inhibition of MAPK by a dominantly negative construct of Ras prevented netrin-1-induced axon branching (Fig. 9I). Interestingly, branches induced by MAPK appeared to have fewer secondary and tertiary branches than those elicited by netrin-1 and by CaMKII overexpression. These results suggest that the MAPK pathway activated by netrin-1 plays an important role in axon branching but not in axon outgrowth.

\section{Discussion}

Despite the essential role of axon branching in establishing CNS connectivity, surprisingly little is known about the mechanisms by which branching is regulated (Dent et al., 2003; Kornack and Giger, 2005). In the present study, we show that netrin-1 induces rapid and extensive cortical axon branching through intracellular $\mathrm{Ca}^{2+}$ signaling pathways. Application of netrin-1-induced repetitive $\mathrm{Ca}^{2+}$ transients in the axon or increased the frequency of endogenous $\mathrm{Ca}^{2+}$ transients. $\mathrm{Ca}^{2+}$ transients could be evoked in the entire axon or could begin in localized axon regions and 
spread throughout the axon. Experimental reduction of $\mathrm{Ca}^{2+}$ levels revealed that $\mathrm{Ca}^{2+}$ signaling is essential for netrin-1induced axon branching, and the use of specific receptor blockers revealed that release from intracellular stores is a major source of this $\mathrm{Ca}^{2+}$ activity. We imaged netrin-1-induced $\mathrm{Ca}^{2+}$ transients in localized regions of the axon, and remarkably, we found that within minutes of netrin-1 application, new branches protruded from those regions of the axon showing highfrequency $\mathrm{Ca}^{2+}$ transients. Thus, for the first time, we show that induction of highfrequency calcium transients along the axon can promote rapid development of branches. In additional experiments, we explored the downstream targets of $\mathrm{Ca}^{2+}$ signaling involved in axon branching. Using a variety of approaches involving pharmacological inhibition, overexpression, transfection with mutagenized constructs, and RNAi knockdown, we found that CaMKII and MAPKs play a major role in axon branching, which is consistent with the sensitivity of these kinases to changes in the frequency $\mathrm{Ca}^{2+}$ transients. Interestingly, CaMKII promotes axon outgrowth as well as axon branching, whereas MAPK affects only axon branching but not axon outgrowth. Together, this study reveals novel mechanisms, whereby netrin-1 $\mathrm{Ca}^{2+}$ signaling promotes cortical axon branching independent of axon outgrowth. Netrin-1 has been shown to attract cortical axons in vivo (Serafini et al., 1996) and directed growth and branching of cortical axons by netrin-1 has also been demonstrated in explant cocultures (Richards et al., 1997). Although the role of netrin-1 in cortical axon branching in vivo is not known, it is possible that similar calcium signaling mechanisms elicited by target-derived cues such as netrin-1 may promote branching to appropriate targets at specific locations along the axon.

\section{Calcium signaling in axon outgrowth and branching}

Studies of axonal development have revealed diverse roles for calcium signaling (Kater and Mills, 1991; Letourneau et al., 1994; Gomez and Spitzer, 2000; Berridge et al., 2003; Henley and Poo, 2004). In the Xenopus spinal cord, axon outgrowth is regulated by frequencies of $\mathrm{Ca}^{2+}$ transients in the growth cone $(\mathrm{Gu}$ and Spitzer, 1995; Gomez and Spitzer, 1999). Suppression of $\mathrm{Ca}^{2+}$ transients accelerates axon outgrowth, whereas imposing $\mathrm{Ca}^{2+}$ transients slows growth cone advance (Gomez and Spitzer, 1999). Previously, we found that, in dissociated cortical neurons, spontaneous global $\mathrm{Ca}^{2+}$ transients regulate axon outgrowth in a frequency-dependent manner (Tang et al., 2003). When $\mathrm{Ca}^{2+}$ activity was silenced by L-type channel blockers, axons accelerated their advance. Thus, global $\mathrm{Ca}^{2+}$ transients appear to regulate cortical axon outgrowth in a frequency-dependent manner.

In contrast, local $\mathrm{Ca}^{2+}$ transients appear to be involved in axon guidance. Spatially restricted elevation of intracellular $\mathrm{Ca}^{2+}$ concentration in the growth cone induced by guidance cues has been shown to initiate turning behaviors in the direction of higher calcium (Zheng et al., 1994; Hong et al., 2000; Zheng, 2000). In response to gradients of netrin-1, intracellular $\mathrm{Ca}^{2+}$ concentrations are elevated in the growth cone to regulate changes in guidance behaviors (Hong et al., 2000; Ming et al., 2002; Nishiyama et al., 2003). Attractive turning was preceded by protrusion of lammelipodia and filopodia (Zheng, 2000). These effects involve relative $\mathrm{Ca}^{2+}$ concentrations that form a gradient across the growth cone (Zheng et al., 1994; Hong et al., 2000; Henley and Poo, 2004). Induction of localized elevations in $\mathrm{Ca}^{2+}$ concentration also promotes protrusion of filopodia from growth cones (Silver et al., 1990; Davenport and Kater, 1992; Gomez et al., 2001) and from axons (Lau et al., 1999). Our results show that, within minutes, axon branches develop from regions of the axon expressing repetitive high-frequency $\mathrm{Ca}^{2+}$ transients that persist up to $1 \mathrm{~h}$ in response to continuous netrin-1 application. In contrast to artificially imposed $\mathrm{Ca}^{2+}$ elevation, we used a relevant guidance cue to induce $\mathrm{Ca}^{2+}$ transients that reflect the physiological responses of the cortical neuron in branch formation. For this reason, we chose not to elevate calcium by localized application of nonphysiological reagents such as $\mathrm{KCl}$, which transiently elevates levels of intracellular calcium ( $\mathrm{Gu}$ and Spitzer, 1995) rather than evoking prolonged calcium transients or increasing frequencies of calcium transients, as does netrin-1. It might also be difficult to use localized electrical stimulation to impose calcium transients of frequencies optimal for the promotion of axon branching. Although previous studies of axon guidance have emphasized the role of local elevations in levels of intracellular $\mathrm{Ca}^{2+}$ during growth cone turning (Henley and Poo, 2004; Wen et al., 2004), we demonstrate here how axon branching is dependent on increases in the frequency of localized $\mathrm{Ca}^{2+}$ transients. 


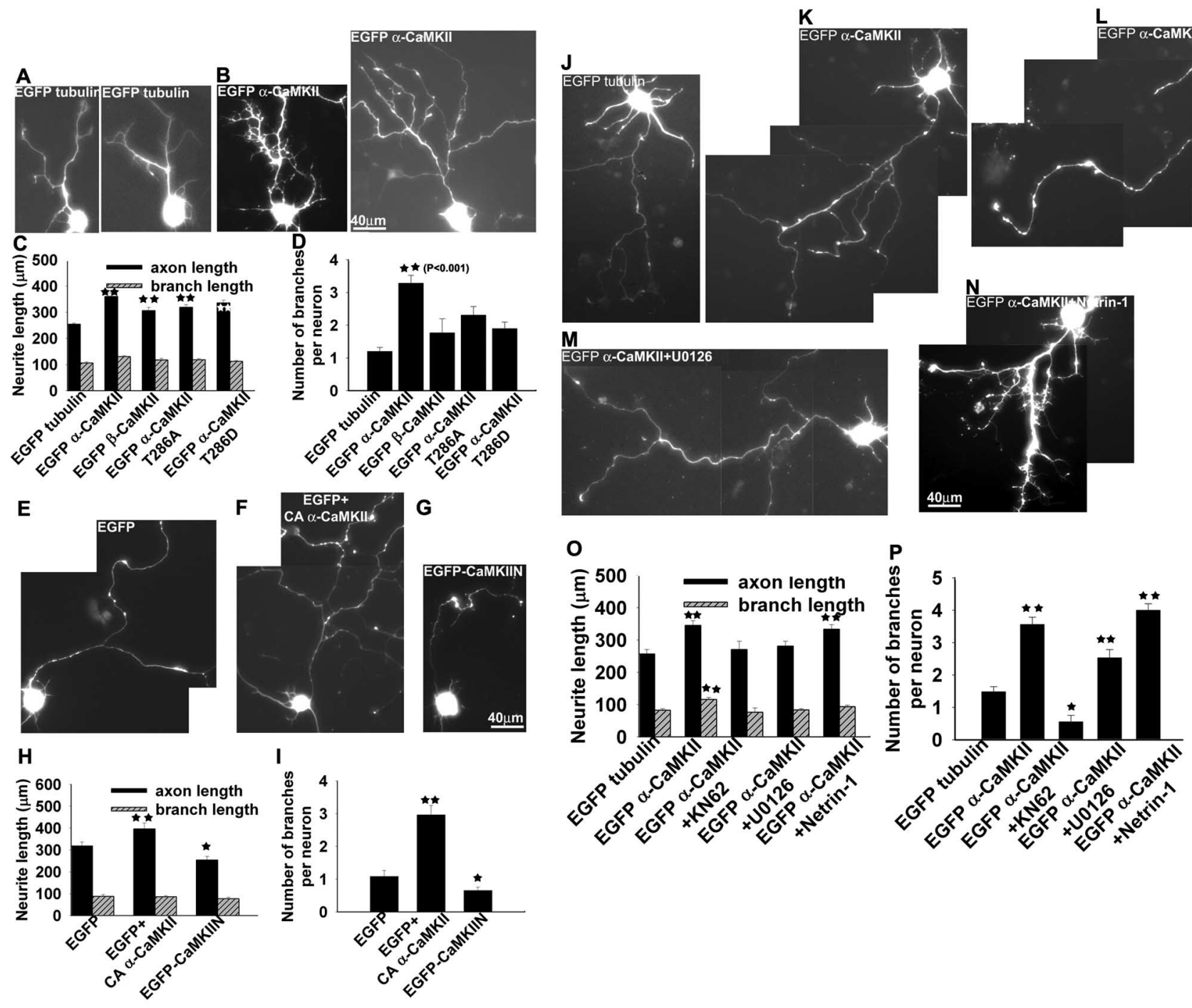

Figure 7. Overexpression of $\alpha$ CaMKII promotes axon branching and increases axon length. $\boldsymbol{A}, \boldsymbol{B}$, Examples of neurons transfected with EGFP-tubulin $(\boldsymbol{A})$ and EGFP- $\alpha$ CaMKII $(\boldsymbol{B})$. Neurons overexpressing $\alpha$ CaMKII develop highly branched arbors similar to those after netrin- 1 treatment. $C, D$, Bar graphs comparing axon length and branch length $(C)$ and number of branches per neuron (D) in neurons overexpressing tubulin, $\alpha$ CaMKII, $\beta$ CAMKII, and two mutant forms of $\alpha$ CaMKII (T286A and T286D) mutated at the threonine 286 autophosphorylation site. Overexpression of wild-type $\alpha$ CaMKII alone promotes axon branching. $\boldsymbol{E}$-G, Examples of neurons transfected with EGFP alone (E), EGFP and a constitutively active (CA) mutant $\alpha$ CaMKII (F), and EGFP-CaMKIIKIIN, a CaMKII inhibitory protein $(\boldsymbol{G}) . \boldsymbol{H}, \boldsymbol{I}$, Bar graphs comparing axon length and branch length $(\boldsymbol{H})$ and number of branches per neuron $(\boldsymbol{I})$ in all conditions. $J-N$, Examples of neurons transfected with EGFP-tubulin $(\boldsymbol{J})$, EGFP- $\alpha$ CaMKII $(\boldsymbol{K})$, and EGFP- $\alpha$ CaMKII with addition of KN $62(\boldsymbol{L})$, U0126 $(\boldsymbol{M})$, or netrin- $\mathbf{1}(\boldsymbol{N}) . \mathbf{0}, \boldsymbol{P}$, Bar graphs comparing axon length and branch length $(\boldsymbol{O})$ and number of branches per neuron in all conditions. KN62 completely abolishes the branch-promoting effects of $\alpha$ CaMKII in contrast to partial reduction with U0126, and netrin-1 slightly enhances the branching effects of $\alpha$ CaMKII.

\section{Downstream targets of calcium signaling during axon branching}

What features of $\mathrm{Ca}^{2+}$ signaling activate specific downstream targets? Changes in the frequency of $\mathrm{Ca}^{2+}$ transients are known to activate genes for different neurotransmitters (Gomez and Spitzer, 2000; Borodinsky et al., 2004). Different frequencies as well as patterns of $\mathrm{Ca}^{2+}$ transients may also target different kinases and phosphatases in the cytoplasm to elicit different cellular responses (Tomida at al., 2003), and specific cellular events may be regulated by calcium transients at optimal frequencies (Eshete and Fields, 2001). $\mathrm{Ca}^{2+}$ oscillations are self renewing, repetitive, and persistent. Moreover, the mechanism of frequency modulation to decode information from calcium signals has advantages over mechanisms based on changes in amplitude of $\mathrm{Ca}^{2+}$ concentration alone (Cullen and Lockyer, 2002), because discrete $\mathrm{Ca}^{2+}$ oscillations can be more easily distinguished by calcium decoders such as CaMKII (De Koninck and Schulman, 1998) and PKC (Oancea and Meyer, 1998). Furthermore, as shown here, discrete $\mathrm{Ca}^{2+}$ oscillations can be shown to temporally precede and spatially coincide with discrete axon branching events.

Because calcium is a ubiquitous intracellular signal that participates in many diverse cellular processes, it has been difficult to identify specific downstream targets activated by calcium in axon growth and guidance (Lautermilch and Spitzer, 2000; Henley and Poo, 2004). Calcium may activate different targets to elicit opposing effects on the axon, such as inhibiting axon outgrowth but promoting the development of new branches. We identified $\alpha$ CaMKII as a major target of calcium signaling, which is essential for netrin-1-induced axon branching. Previous reports have shown that CaMKII plays an important role in neurite outgrowth and growth cone motility (Goshima et al., 1993) as well as growth 


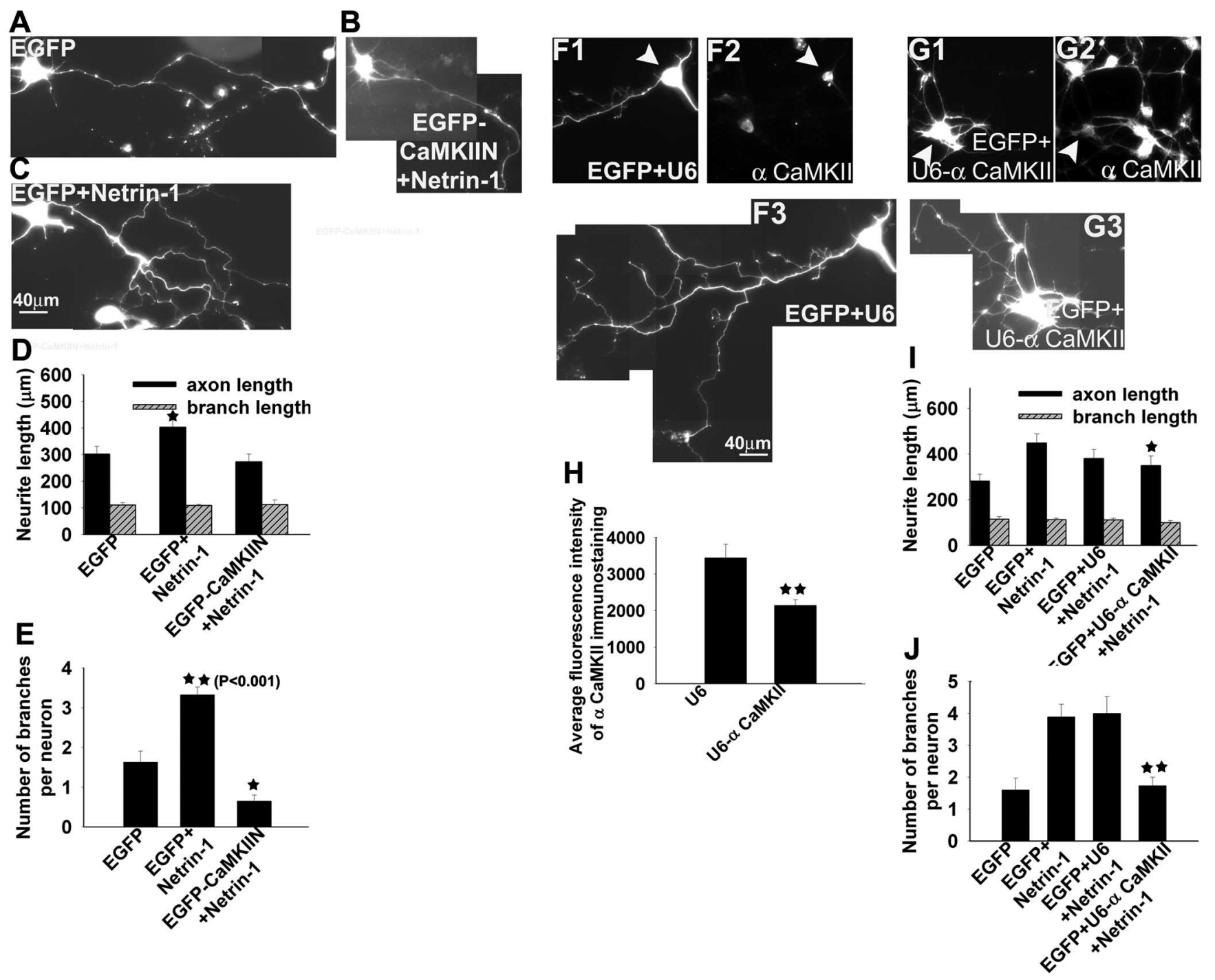

Figure 8. Molecular and RNAi knockdown of CAMKII abolishes netrin-1-induced axon branching. $\boldsymbol{A}-\boldsymbol{C}$, Examples of neurons transfected EGFP $(\boldsymbol{A})$, EGFP and treated with netrin-1 $(\boldsymbol{B})$, and EGFP-CaMKIIN and treated with netrin-1.D, $\boldsymbol{E}$, Bar graphs comparing axon length and branch length $(\boldsymbol{D})$ and number of branches per neuron $(\boldsymbol{E})$ in all conditions. Netrin- 1 cannot promote branching in neurons overexpressing the inhibitory CaMKIIN protein. $\boldsymbol{F 1}-\boldsymbol{F 3}$, Example of a neuron (arrowheads) at $1 \mathrm{~d}$ in culture cotransfected with EGFP and a control DNA vector U6 (F1) and immunostained with an antibody to $\alpha$ CaMKII at $3 \mathrm{~d}$ in culture $(\boldsymbol{F 2})$. This same neuron, when stimulated with netrin-1, is able to branch extensively $(\boldsymbol{F} 3)$. Thus, the control U6 vector does not affect levels of CaMKII or the ability of the axon to branch in the presence of netrin-1. G1-G3, Example of a neuron (arrowheads) at $1 \mathrm{~d}$ in culture cotransfected with EGFP and a U6 - $\alpha$ CaMKII RNAi vector (G1). The same neuron is shown in a different fluorescent channel (G2) demonstrating immunostaining with an antibody to $\alpha$ CaMKII at $3 \mathrm{~d}$ in culture. The neuron (arrowhead) after RNAi knockdown of CaMKII has reduced levels of $\alpha$ CaMKII compared with the neighboring cells and concomitantly few axon branches (G3) when stimulated with netrin-1. $\boldsymbol{H}$, Bar graph comparing fluorescence intensity of $\alpha$ CaMKII immunostaining in neurons transfected with the control U6 vector or the RNAi U6 - $\alpha$ CaMKII. $I, J$, Bar graphs comparing axon length and branch length $(I)$ and number of branches per neuron $(J)$ in all conditions.

cone guidance (Zheng et al., 1994; Kuhn et al., 1998; Wen et al., 2004). Wen et al. (2004) showed that pharmacological inhibition of CaMKII prevented netrin-1-induced repulsive growth cone turning and that local high increases in the amplitude of $\mathrm{Ca}^{2+}$ signals induced turning behaviors. Our results also show the involvement of CaMKII in the netrin-1 signaling pathway. However, we demonstrate a signaling mechanism that is dependent on the induction of high-frequency $\mathrm{Ca}^{2+}$ transients for axon branching. Others have found that CaMKI, but not CaMKII, promotes axon extension and growth cone motility (Wayman et al., 2004). Similarly, both positive (Fink et al., 2003; Jourdain et al., 2003; Gaudilliere et al., 2004) and inhibitory (Wu and Cline, 1998; Redmond et al., 2002) effects for CaMKII have been described for dendritic growth and branching. Although the reasons for these discrepancies are unclear, the present results ob- tained with a variety of techniques including pharmacological and molecular CaMKII inhibitors, overexpression of EGFP CaMKII, and RNAi knockdown provide strong evidence that $\alpha$ CaMKII is essential for netrin-1-induced cortical axon branching. Our results also suggest that CaMKII has a global effect on axon outgrowth, because CaMKII increases axon length as well as branching either with or without application of netrin-1. MAPKs have also been shown to influence netrin-1-induced axon guidance (Forcet et al., 2002; Ming et al., 2002; Campbell and Holt, 2003). Consistent with these studies, our results show that MAPK specifically promotes axon branching without affecting axon outgrowth. However, it is not clear why, in contrast to our results for cortical neurons, spinal commissural neurons require MAPK signaling for netrin-1-mediated axon outgrowth (Forcet et al., 2002). Interestingly, overexpression of CaMKII in the presence of 




Figure 9. MAPK overexpression promotes and pharmacological or molecular inhibition of MAPK prevents netrin-1-induced axon branching without affecting axon outgrowth. $A$, Examples of neurons treated with BSA (control), netrin, and the MAPK inhibitors U0126 and PD98059 in combination with netrin and the CAMKII inhibitor KN62. B, C, Bar graphs comparing axon length and branch length $(\boldsymbol{B})$ and number of branches per neuron $(\boldsymbol{C})$ in all conditions. Both MAPK inhibitors prevent netrin-1-induced branching, and the combination of U0126 with KN62 severely reduces axon outgrowth and abolishes netrin-1-induced branching. $\boldsymbol{D}$-I, Examples of neurons transfected with EGFP alone (D), EGFP and a constitutively active form of MEK1 (E), myc-tagged Erk2-MEK1-LA $(\boldsymbol{F})$, a control myc-tagged Erk2-MEK1 (G), EGFP with the addition of netrin-1 $(\boldsymbol{H})$, and EGFP with a dominant-negative form of Ras (RasN17) in the presence of netrin-1 (I). J-M, Bar graphs comparing axon length and branch length $(J, L)$ and number of branches per neuron $(\boldsymbol{K}, \boldsymbol{M})$ in all conditions.

MAPK inhibitors greatly reduces axon branching (Fig. $7 \mathrm{M}, P$ ), suggesting that CaMKII can activate MAPKs (Chen et al., 1998). Interactions between these signaling pathways are not well understood. In preliminary experiments, however, we found that constitutively active CaMKII activates MAPK as determined by immunostaining with phospho-MAPK (our unpublished results). Second, we found that the CaMKII inhibitor KN62 suppresses netrin-1-induced phosphorylation of MAPK. Both results suggest that CaMKII can activate MAPK signaling. However, molecular mechanisms of interaction between these two signaling pathways are beyond the scope of this study.

Effects of calcium signaling pathways on the cytoskeleton Ultimately, signaling pathways activated by guidance cues must converge on the actin-microtubule cytoskeleton to induce growth cone turning behaviors or axon branching (Dent and Gertler, 2003). Cortical axon branching has been shown to require dynamic actin-microtubule interactions (Dent and Kalil, 2001), and netrin-1 elicits filopodial protrusion through actin polymerization (Dent et al., 2004; Lebrand et al., 2004). Netrin-1 is known to activate Rho GTPases, which regulate actin polymerization (Li et al., 2002; Shekarabi and Kennedy, 2002), and also activate MAP $1 \mathrm{~B}$, which is thought to play a role in actin-microtubule interactions (Del Rio et al., 2004). Thus, netrin-1-induced calcium signaling could influence regulators of cytoskeletal dynamics required for axon branching. We found that CaMKII is a major target of netrin-1-induced calcium signaling. Studies of regulation of dendritic morphology and plasticity suggest that CaMKII may be an important link to the cytoskeleton. In dendritic filopodia, resembling spines, $\alpha$ CaMKII was shown to inter- 
act with $\beta$ CaMKII and translocate to dendritic spines, thereby targeting CaMKII to the actin cytoskeleton (Shen et al.,1998). This is consistent with the role of CaMKII in stabilizing dendritic arbors (Wu and Cline, 1998). Although these studies do not identify an exact role for CaMKII in regulating actin polymerization, there is evidence (Chen et al., 2003) that CaMKII can activate cdc42, which polymerizes actin during filopodia formation. The repetitive calcium transients induced by netrin-1 described here could provide a mechanism for translocating CaMKII to actin filaments in nascent axonal filopodia. In the future, it will be important to understand exactly how major targets of calcium signaling, such as CaMKII and MAPK, regulate cytoskeletal dynamics required for development of new branches.

\section{References}

Berridge MJ, Bootman MD, Roderick HL (2003) Calcium signalling: dynamics, homeostasis and remodelling. Nat Rev Mol Cell Biol 4:517-529.

Borodinsky LN, Root CM, Cronin JA, Sann SB, Gu X, Spitzer NC (2004) Activity-dependent homeostatic specification of transmitter expression in embryonic neurons. Nature 429:523-530.

Campbell DS, Holt CE (2003) Apoptotic pathway and MAPKs differentially regulate chemotropic responses of retinal growth cones. Neuron 37:939-952.

Chang BH, Mukherji S, Soderling TR (1998) Characterization of a calmodulin kinase II inhibitor protein in brain. Proc Natl Acad Sci USA 95:10890-10895.

Chen HJ, Rojas-Soto M, Oguni A, Kennedy MB (1998) A synaptic RasGTPase activating protein (p135 SynGAP) inhibited by CaM kinase II. Neuron 20:895-904.

Chen N, Furuya S, Doi H, Hashimoto Y, Kudo Y, Higashi H (2003) Ganglioside/calmodulin kinase II signal inducing cdc42-mediated neuronal actin reorganization. Neuroscience 120:163-176.

Colavita A, Tessier-Lavigne M (2003) A neurexin-related protein, BAM-2, terminates axonal branches in C. elegans. Science 302:293-296.

Cullen PJ, Lockyer PJ (2002) Integration of calcium and Ras signalling. Nat Rev Mol Cell Biol 3:339-348.

Davenport RW, Kater SB (1992) Local increases in intracellular calcium elicit local filopodial responses in Helisoma neuronal growth cones. Neuron 9:405-416.

De Koninck P, Schulman H (1998) Sensitivity of CaM kinase II to the frequency of $\mathrm{Ca}^{2+}$ oscillations. Science 279:227-230.

Del Rio JA, Gonzalez-Billault C, Urena JM, Jimenez EM, Barallobre MJ, Pascual M, Pujadas L, Simo S, La Torre A, Wandosell F, Avila J, Soriano E (2004) MAP1B is required for Netrin 1 signaling in neuronal migration and axonal guidance. Curr Biol 14:840-850.

Dent EW, Gertler FB (2003) Cytoskeletal dynamics and transport in growth cone motility and axon guidance. Neuron 40:209-227.

Dent EW, Kalil K (2001) Axon branching requires interactions between dynamic microtubules and actin filaments. J Neurosci 21:9757-9769.

Dent EW, Tang F, Kalil K (2003) Axon guidance by growth cones and branches: common cytoskeletal and signaling mechanisms. The Neuroscientist 9:343-353.

Dent EW, Barnes AM, Tang F, Kalil K (2004) Netrin-1 and semaphorin 3A promote or inhibit cortical axon branching, respectively, by reorganization of the cytoskeleton. J Neurosci 24:3002-3012.

Dickson BJ (2002) Molecular mechanisms of axon guidance. Science 298:1959-1964.

Dolmetsch RE, Pajvani U, Fife K, Spotts JM, Greenberg ME (2001) Signaling to the nucleus by an L-type calcium channel-calmodulin complex through the MAP kinase pathway. Science 294:333-339.

Eshete F, Fields RD (2001) Spike frequency decoding and autonomous activation of $\mathrm{Ca}^{2+}$-calmodulin-dependent protein kinase II in dorsal root ganglion neurons. J Neurosci 21:6694-6705.

Fink CC, Bayer KU, Myers JW, Ferrell Jr JE, Schulman H, Meyer T (2003) Selective regulation of neurite extension and synapse formation by the beta but not the alpha isoform of CaMKII. Neuron 39:283-297.

Forcet C, Stein E, Pays L, Corset V, Llambi F, Tessier-Lavigne M, Mehlen P (2002) Netrin-1-mediated axon outgrowth requires deleted in colorectal cancer-dependent MAPK activation. Nature 417:443-447.

Gaudilliere B, Konishi Y, de la Iglesia N, Yao G, Bonni A (2004) A CaMKII-
NeuroD signaling pathway specifies dendritic morphogenesis. Neuron 41:229-241.

Gomez TM, Spitzer NC (1999) In vivo regulation of axon extension and pathfinding by growth-cone calcium transients. Nature 397:350-355.

Gomez TM, Spitzer NC (2000) Regulation of growth cone behavior by calcium: new dynamics to earlier perspectives. J Neurobiol 44:174-183.

Gomez TM, Robles E, Poo M, Spitzer NC (2001) Filopodial calcium transients promote substrate-dependent growth cone turning. Science 291:1983-1987.

Goshima Y, Ohsako S, Yamauchi T (1993) Overexpression of $\mathrm{Ca}^{2+} /$ calmodulin-dependent protein kinase II in Neuro2a and NG108-15 neuroblastoma cell lines promotes neurite outgrowth and growth cone motility. J Neurosci 13:559-567.

Gu X, Spitzer NC (1995) Distinct aspects of neuronal differentiation encoded by frequency of spontaneous $\mathrm{Ca}^{2+}$ transients. Nature 375:784-787.

Halloran MC, Kalil K (1994) Dynamic behaviors of growth cones extending in the corpus callosum of living cortical brain slices observed with video microscopy. J Neurosci 14:2161-2177.

Henley J, Poo MM (2004) Guiding neuronal growth cones using $\mathrm{Ca}^{2+}$ signals. Trends Cell Biol 14:320-330.

Hong K, Nishiyama M, Henley J, Tessier-Lavigne M, Poo M (2000) Calcium signalling in the guidance of nerve growth by netrin-1. Nature 403:93-98.

Hudmon A, Schulman H (2002) Neuronal $\mathrm{CA}^{2+} /$ calmodulin-dependent protein kinase II: the role of structure and autoregulation in cellular function. Annu Rev Biochem 71:473-510.

Jourdain P, Fukunaga K, Muller D (2003) Calcium/calmodulin-dependent protein kinase II contributes to activity-dependent filopodia growth and spine formation. J Neurosci 23:10645-10649.

Kater SB, Mills LR (1991) Regulation of growth cone behavior by calcium. J Neurosci 11:891-899.

Kornack DR, Giger RJ (2005) Probing microtubule + TIPs: regulation of axon branching. Curr Opin Neurobiol 15:58-66.

Kuhn TB, Williams CV, Dou P, Kater SB (1998) Laminin directs growth cone navigation via two temporally and functionally distinct calcium signals. J Neurosci 18:184-194.

Lau PM, Zucker RS, Bentley D (1999) Induction of filopodia by direct local elevation of intracellular calcium ion concentration. J Cell Biol 145:1265-1275.

Lautermilch NJ, Spitzer NC (2000) Regulation of calcineurin by growth cone calcium waves controls neurite extension. J Neurosci 20:315-325.

Lebrand C, Dent EW, Strasser GA, Lanier LM, Krause M, Svitkina TM, Borisy GG, Gertler FB (2004) Critical role of Ena/VASP proteins for filopodia formation in neurons and in function downstream of netrin-1. Neuron 42:37-49.

Letourneau PC, Snow DM, Gomez TM (1994) Regulation of growth cone motility by substratum bound molecules and cytoplasmic $\left[\mathrm{Ca}^{2+}\right]$. Prog Brain Res 103:85-98.

Li X, Saint-Cyr-Proulx E, Aktories K, Lamarche-Vane N (2002) Racl and Cdc42 but not RhoA or Rho kinase activities are required for neurite outgrowth induced by the Netrin-1 receptor DCC (deleted in colorectal cancer) in N1E-115 neuroblastoma cells. J Biol Chem 277:15207-15214.

Ming GL, Wong ST, Henley J, Yuan XB, Song HJ, Spitzer NC, Poo MM (2002) Adaptation in the chemotactic guidance of nerve growth cones. Nature 417:411-418.

Ng J, Nardine T, Harms M, Tzu J, Goldstein A, Sun Y, Dietzl G, Dickson BJ, Luo L (2002) Rac GTPases control axon growth, guidance and branching. Nature 416:442-447.

Nishiyama M, Hoshino A, Tsai L, Henley JR, Goshima Y, Tessier-Lavigne M, Poo MM, Hong K (2003) Cyclic AMP/GMP-dependent modulation of $\mathrm{Ca}^{2+}$ channels sets the polarity of nerve growth-cone turning. Nature 423:990-995.

Oancea E, Meyer T (1998) Protein kinase C as a molecular machine for decoding calcium and diacylglycerol signals. Cell 95:307-318.

O'Leary DD, Bicknese AR, De Carlos JA, Heffner CD, Koester SE, Kutka LJ, Terashima T (1990) Target selection by cortical axons: alternative mechanisms to establish axonal connections in the developing brain. Cold Spring Harb Symp Quant Biol 55:453-468.

Redmond L, Kashani AH, Ghosh A (2002) Calcium regulation of dendritic growth via CaM kinase IV and CREB-mediated transcription. Neuron 34:999-1010.

Richards LJ, Koester SE, Tuttle R, O'Leary DD (1997) Directed growth of 
early cortical axons is influenced by a chemoattractant released from an intermediate target. J Neurosci 17:2445-2458.

Schwab ME (2002) Increasing plasticity and functional recovery of the lesioned spinal cord. Prog Brain Res 137:351-359.

Serafini T, Colamarino SA, Leonardo ED, Wang H, Beddington R, Skarnes WC, Tessier-Lavigne M (1996) Netrin-1 is required for commissural axon guidance in the developing vertebrate nervous system. Cell 87:1001-1014.

Shekarabi M, Kennedy TE (2002) The netrin-1 receptor DCC promotes filopodia formation and cell spreading by activating Cdc42 and Rac1. Mol Cell Neurosci 19:1-17.

Shen K, Teruel MN, Subramanian K, Meyer T (1998) CaMKIIbeta functions as an F-actin targeting module that localizes CaMKIIalpha/beta heterooligomers to dendritic spines. Neuron 21:593-606.

Silver RA, Lamb AG, Bolsover SR (1990) Calcium hotspots caused by L-channel clustering promote morphological changes in neuronal growth cones. Nature 343:751-754.

Soderling TR, Chang B, Brickey D (2001) Cellular signaling through multifunctional $\mathrm{Ca}^{2+} /$ calmodulin-dependent protein kinase II. J Biol Chem 276:3719-3722.

Sui G, Soohoo C, Affar el B, Gay F, Shi Y, Forrester WC (2002) A DNA vector-based RNAi technology to suppress gene expression in mammalian cells. Proc Natl Acad Sci USA 99:5515-5520.

Szebenyi G, Callaway JL, Dent EW, Kalil K (1998) Interstitial branches de- velop from active regions of the axon demarcated by the primary growth cone during pausing behaviors. J Neurosci 18:7930-7940.

Tang F, Dent EW, Kalil K (2003) Spontaneous calcium transients in developing cortical neurons regulate axon outgrowth. J Neurosci 23:927-936.

Tomida T, Hirose K, Takizawa A, Shibasaki F, Iino M (2003) NFAT functions as a working memory of $\mathrm{Ca}^{2+}$ signals in decoding $\mathrm{Ca}^{2+}$ oscillation. EMBO J 22:3825-3832.

Vaillant AR, Mazzoni I, Tudan C, Boudreau M, Kaplan DR, Miller FD (1999) Depolarization and neurotrophins converge on the phosphatidylinositol 3-kinase-Akt pathway to synergistically regulate neuronal survival. J Cell Biol 146:955-966.

Wayman GA, Kaech S, Grant WF, Davare M, Impey S, Tokumitsu H, Nozaki N, Banker G, Soderling TR (2004) Regulation of axonal extension and growth cone motility by calmodulin-dependent protein kinase I. J Neurosci 24:3786-3794.

Wen Z, Guirland C, Ming GL, Zheng JQ (2004) A CaMKII/calcineurin switch controls the direction of $\mathrm{Ca}(2+)$-dependent growth cone guidance. Neuron 43:835-846.

Wu GY, Cline HT (1998) Stabilization of dendritic arbor structure in vivo by CaMKII. Science 279:222-226.

Zheng JQ (2000) Turning of nerve growth cones induced by localized increases in intracellular calcium ions. Nature 403:89-93.

Zheng JQ, Felder M, Connor JA, Poo MM (1994) Turning of nerve growth cones induced by neurotransmitters. Nature 368:140-144. 